\title{
Calidad de vida relacionada con la salud oral, características socioeconómicas y de comportamiento de los pacientes fisurados menores de 7 años
}

\author{
Ana Carolina Silva Cordeiro ${ }^{1}$; \\ Isabel Cristina Olegário da Costa ${ }^{2}$; \\ Carmela Rampazzo Bresolin ${ }^{2}$; \\ José Carlos Pettorossi Imparato ${ }^{3}$
}

\section{Resumen}

Introducción: Labio y paladar fisurado es una malformación congénita en la región del labio y/o paladar, pudiendo ser uni o bilateral, causada por la falta de cierre de dichas estructuras, su gravedad puede variar y afectar los hábitos y la calidad de vida en relación con la salud oral. Objetivo: El objetivo de este estudio es evaluar la calidad de vida relacionada con la salud oral y las características socio-conductuales de pacientes fisurados menores de 7 años. Materiales y Métodos: Después de la aprobación del comité de ética, el estudio se llevó a cabo en un centro de atención clínica de los pacientes con dicha alteracion, y la muestra de conveniencia consistió en 19 pacientes cuyos cuidadores aceptaron participar y responder a los cuestionarios solicitados. Un cuestionario para medir la calidad de vida en relación con la salud oral se aplicó a los res- ponsables. Las variables socioeconómicas se recolectaron a través de un cuestionario específico utilizado previamente, así como las preguntas sobre el miedo y el cuidado de la higiene bucal de los niños. Los datos se analizaron de manera descriptiva. Resultados: La mayoría de los pacientes pertenecían al sexo masculino y la edad media fue de 3,9 años. Gran parte de los cuidadores reportó dificultades y el miedo para la realización de la higiene bucal y alimentación de los niños. En cuanto a la calidad de vida se percibió un mayor impacto en la sesión del niño que en de la familia. Conclusión: Se puede concluir que los pacientes menores de 7 años con labio y paladar fisurados pueden tener dificultades en la limpieza y la alimentación, así como los cambios en la calidad de vida relacionada con la salud oral.

Palabras Clave: Niño. Calidad de vida. Salud bucal. Labio y paladar fisurado.

\footnotetext{
1. Especialista en Odontopediatria. Facultad de Odontología São Leopoldo Mandic, São Paulo, Brasil.

2. Doctoranda en Odontopediatria. Facultad de Odontología. Universidad de São Paulo, São Paulo, Brasil.

3. Profesor Titular de la disciplina de Odontopediatría. Facultad de Odontología. Universidad de São Paulo, São Paulo, Brasil.
} 
Artigo Original

\section{Qualidade de vida com relação à saúde bucal, características socioeconômicas e comportamentais de pacientes fissurados menores de 7 anos}

\section{Resumo}

Introdução: A fissura labiopalatina é uma má formação congênita na região do lábio e/ou palato, ocasionada pelo não fechamento de tais estruturas, sua gravidade pode variar e afetar a alimentação, fonética e a qualidade de vida com relação à saúde bucal dos pacientes. Objetivo: O objetivo desse estudo foi avaliar a qualidade de vida com relação à saúde bucal e características socio-comportamentais de pacientes com fissuras labio palatinas menores de 7 anos. Materiais e Métodos: Após aprovação do comitê é ética, o estudo foi realizado em uma clínica de atendimento de pacientes fissurados, e a amostra de conveniência foi composta por 19 pacientes cujos cuidadores aceitaram participar do estudo e responderam aos questionários solicitados. Para mensurar a qualidade de vida com relação à saúde bucal foi aplicado o questionário Early Childhood Oral Health Impact Scale aos responsáveis. As variáveis socioeconômicas foram coletadas através de um questionário específico para o estudo, além de perguntas sobre o medo e os cuidados com a higiene bucal das crianças. Os dados foram analisados descritivamente. Resultados: A maioria dos pacientes pertenceram ao gênero masculino e a média de idade foi de 3,9 anos. Grande parte dos cuidadores relataram dificuldade e medo para fazer a higienização bucal das crianças e dificuldade dos mesmos para se alimentarem. Com relação à qualidade de vida foi percebido um impacto maior na sessão da criança que no da família. Conclusão: Pode-se concluir que pacientes menores de 7 anos com fissuras labio palatinas podem ter dificuldade na limpeza e alimentação bem como alterações na qualidade de vida com relação à saúde bucal.

Palavras-chave: Criança. Qualidade de vida. Saúde bucal. Fenda labial.

Original article

\section{Oral health related quality of life, socioeconomic and behavioral characteristics in children under 7-years old with cleft lip and palate.}

\section{Summary}

Introduction: Cleft lip and/or cleft palate is a congenital malformation in the lip region and/ or palate, caused by the lack of closure in such structures. Severity can vary and affect the habits and the oral health related quality of life in this patients. Objective: The objective of this 
study is to evaluate the quality of life in relation to oral health and socio-behavioral characteristics of patients under 7-years old with cleft lip and palate. Materials and Methods: After the approval of the ethics committee, the study was conducted at a clinic for patients with cleft lip and/or palate. A convenience sample consisted of 19 patients whose caregivers agreed to participate and answer the requested questionnaires. The Early Childhood Oral Health Impact Scale questionnaire was applied to measure the quality of life in relation to oral health. Socioeconomic variables were collected through a specific questionnaire used in previously studies, as well as questions about

\section{Introducción}

La fisura labio palatina es una malformación congénita que consiste en una apertura en la región del labio y/o velo palatino, ocasionada por la falla en el cierre de tales estructuras. Esto ocurre entre la $4^{\circ}$ y la $12^{\circ}$ semana de vida intrauterina, siendo que la gravedad puede variar. Las fisuras pueden ser uni o bilaterales, cicatriz labial o úvula bífida y sus formas más severas son fisuras completas del labio y velo palatino. ${ }^{1}$

La fisura es una de las anomalías craneofaciales más prevalentes, siendo que, de cada 700 niños nacidos, 1 tiene la malformación. ${ }^{2}$ Estas malformaciones pueden hacer que los niños tengan una capacidad disminuida para alimentarse, ocasionando problemas para ganar peso y mala nutrición. ${ }^{3}$

Los pacientes fisurados pueden estar afectados por una combinación de varias diferencias faciales: disturbios de la dentición y del crecimiento de los maxilares; así como de la deglución, del fear and care for oral hygiene of children. Data were analyzed descriptively. Results: Most patients belonged to the male and the average age was 3.9 years. Most caregivers reported difficulty and fear to perform oral hygiene of children and difficulty to feed them. Regarding the quality of life, a greater impact on the child than in the family was found. Conclusion: It can be concluded that patients younger than 7 years of age with clefts lip and palate may have difficulty in cleaning and feeding as well as changes in oral health related quality of life.

Key words: Child. Quality of life. Oral health. Cleft lip.

habla y de la audición. ${ }^{4}$ Además, la región donde ocurre la fisura es altamente visible en el cuerpo y puede provocar comprometimiento estético y psicosocial para estas personas, que afectan su calidad de vida. ${ }^{6}$

La calidad de vida se refiere a una representación multi-dimensional y sensación subjetiva del bienestar e incluye los efectos físicos y psicológicos del tratamiento, así como de la familia y del ambiente. ${ }^{7}$ Para medir la calidad de vida con relación a la salud bucal, frecuentemente se usan indicadores socio dentales como una variable de aproximación de las medidas normativas para evaluar las necesidades de salud de una población dada. ${ }^{8,9}$

La literatura muestra estudios evaluando el impacto de la fisura labiopalatal en la calidad de vida relacionada a la salud bucal en diferentes grupos de edades, especialmente en adolescentes. ${ }^{5}$ Además, los estudios muestran la relación entre condiciones socioeconómicas y condiciones inferiores de la salud bucal en estos pacientes. ${ }^{4,10,11}$ Sin embargo, 
son escasos los relatos en la literatura donde se investigan la calidad de vida en pacientes menores de 7 años.

Debido a esto, el objetivo de este estudio es describir las características socioeconómicas, comportamentales y la calidad de vida con relación a la salud bucal de pacientes menores de 7 años con fisuras labio palatales, a fin de levantar datos de esta población poco investigada.

\section{Material y Método}

\section{Preceptos éticos y muestra}

Este estudio fue sometido al Comité de Ética en investigación de la Facultad de Odontología "São Leopoldo Mandic" y aprobado en sus aspectos éticos y metodológicos de acuerdo con las Directivas establecidas en la Resolución $\mathrm{n}^{\circ} 466$ y complementario del Consejo Nacional de Salud de Brasil (CAAE 52569615.0.0000.5374).

La muestra estuvo formada por pacientes atendidos en la Asociación de los Fisurados Labio Palatales de Sorocaba y región (Afissore) en la ciudad de Sorocaba, São Paulo, Brasil.

Fue elegida una muestra de conveniencia por un estudio transversal y descriptivo. Se invitó a formar parte de la investigación a pacientes de hasta 7 años, con fisuras labio palatales.

\section{Criterios de Elegibilidad}

Fueron incluidos pacientes con fisuras de labio y velo palatino ya sometidos a tratamiento quirúrgico y cuyos padres concordaron y autorizaron la participación por el término de Consenti- miento Libre y Esclarecido. Los sujetos podrían retirarse de la investigación en cualquier etapa.

Los responsables de los pacientes autorizados a participar en la investigación fueron entrevistados en el consultorio odontológico individual por una entrevistadora entrenada.

\section{Cuestionarios}

Para obtener la información de la calidad de vida con relación a la salud bucal y condiciones socioeconómicas, los padres contestaron al cuestionario de edad específico (Early Childhood Oral Health Impact Scale - ECOHIS) ${ }^{12}$ y un cuestionario socioeconómico, utilizado en estudios previos, con preguntas dirigidas a los responsables de los pacientes. La entrevista fue presencial. $^{13}$

La calidad de vida con relación a la salud bucal para el grupo de edad de la indagación se evaluó por medio de un cuestionario específico para la edad en su versión brasileña que es el ECOHIS, que evalúa a los niños de hasta 7 años de edad e incluye una sección de "Impacto en el niño" (síntomas, función, psicología y autoimagen, dominios de interacción social) y una sección de "Impacto en la familia" (dominios función de la familia).

Las respuestas fueron anotadas usando una escala de Likert con opciones de respuesta codificada $0-5(0=$ no, $1=$ raramente, $2=$ a veces, $3=$ frecuentemente, $4=$ muy frecuentemente; $5=$ no sabe). Las puntuaciones del ECOHIS fueron computadas por la suma de las puntuaciones para cada dominio después de la recodificación de todos "no sabe" como falta. Para aquellos con hasta dos respuestas en falta en la sección 
del niño o una respuesta en falta en la sección familia, se atribuyó para los artículos en falta una media de los artículos restantes. Los resultados más altos denotan mayor impacto de las condiciones bucales en la calidad de vida del niño.

\section{Análisis Estadístico}

Los datos fueron analizados utilizando el paquete estadístico Stata 12.0. Se realizó un análisis descriptivo de las variables socioeconómicas y específicas para obtener las características de la muestra y el análisis descriptivo de cada sección del cuestionario ECOHIS. Para el análisis se utilizó la media como medida de tendencia céntrica.

\section{Resultados}

Participaron en este estudio 19 pacientes portadores de fisura labio palatinas atendidos en la Asociación de los Fisurados Labio Palatales de Sorocaba y región (Afissore). De estos, la mayoría de los pacientes del sexo masculino $(63.16 \%)$ y la media de edad de los pacientes fue de 3.9 años. Todos los pacientes de raza blanca y sólo uno de ellos no vivía con la madre ni el padre. El ingreso mensual promedio de los participantes fue de $\mathrm{R} \$ 2,163.00$ (variando de $\mathrm{R} \$ 600.00$ a $\mathrm{R} \$ 6,500.00$ ) (Tabla 1).

Los padres de la mayoría de los participantes relataron dificultades tanto para que el niño se alimentase $(63.16 \%)$ como para que se cepillase los dientes $(63.16 \%)$.

En la sección de la calidad de vida del niño con relación a la salud bucal hubo una gran variabilidad de respuestas (0-5), pero la media fue baja si se considera que el ítem 2 corresponde a "a veces". En la sección de "Impacto en la familia", la variabilidad fue más pequeña (0-2) y la media fue de 0.06, o sea, el impacto fue mínimo.

\begin{tabular}{|l|l|}
\hline \multicolumn{1}{|c|}{ Variables } & \multicolumn{1}{|c|}{ N $(\%)$} \\
\hline Sexo & \\
Femenino & $7(36.84)$ \\
Masculino & $12(63.16)$ \\
\hline Edad & $3.9(0.4-7)$ \\
\hline Dificultad para cepillarse & $7(36.84)$ \\
No & $12(63.16)$ \\
Si & \\
\hline Dificultad para alimentarse & $7(36.84)$ \\
No & $12(63.16)$ \\
Si & $2,163(600-6,500)$ \\
\hline Ingreso Familiar ${ }^{\star}$ (Reales Brasileños) & $1.95(0-5)$ \\
\hline ECOHIS & $0.06(0-2)$ \\
Impacto en el niño & \\
Impacto en la Familia & \\
\hline
\end{tabular}

* Media (mín y máx).

Tabla 1. Características muestrales, comportamentales y calidad de vida de pacientes fisurados. 


\section{Discusión}

$\mathrm{Al}$ analizar las características de nuestra muestra, aunque sea una muestra de conveniencia, se puede percibir que la prevalencia fue mayor en los pacientes del sexo masculino (63.16\%). Ese resultado corrobora los estudios previos, donde afirman que las fisuras palatales son más prevalentes en el sexo masculino que en el femenino, ${ }^{1,3,9}$ aunque no se especifican los tipos de fisuras. Sin embargo, en nuestro estudio se incluyeron fisuras labio palatales a fin de mantener los criterios de elegibilidad de la muestra.

La media de edad fue de 3,9 años, siendo que gran parte de los pacientes eran bebés y no poseían dentición primaria completa. A pesar de eso, fue perceptible el recelo relatado por los cuidadores en realizar la higienización de los dientes y de la boca de los pacientes. Esa dificultad fue percibida por Paul y Brandt $(1998)^{14}$ al relatar que la higiene bucal es deficiente en esa población especialmente en la región de la fisura palatal.

La mayoría de los padres expuso dificultad para realizar el cepillado y muchos presentaban recelo y miedo de hacerles daño a los niños. Esa falta de higiene adecuada es indicada como uno de los factores de riesgo para la alta prevalencia de caries en pacientes adolescentes con fisuras. ${ }^{15}$ Autores muestran que los pacientes fisurados tienen una mayor propensión a desarrollar dicha enfermedad, ya que existe gran dificultad de higienización, hecho que fue observado en nuestra muestra. ${ }^{16}$ Esta dificultad puede darse debido a la anatomía variada que permanece después de una cirugía, a la posición apiñada de los dientes y al recelo de hacerles daño a los niños.

Debido a esto, los autores enfatizan la necesidad de mayores investigaciones y estudios futuros con muestras más representativas, así como la necesidad de orientar mejor a los cuidadores para que sepan cómo realizar la correcta higienización desde el nacimiento, a fin de prevenir la aparición de caries y enfermedad periodontal y reducir la alta prevalencia de las mismas. ${ }^{15}$ La Asociación de los Fisurados Labio Palatales de Sorocaba (AFISSORE), a pesar del carácter multidisciplinario, no posee un protocolo para enseñar y entrenar a los cuidadores a higienizar la cavidad bucal de los niños fisurados. Después de levantar los datos y de dialogar con los cuidadores de los niños, quedó clara la necesidad de implementar un abordaje con este foco.

Las fisuras pueden venir acompañadas de anomalías dentales, como dientes en $\mathrm{T}$ o $\mathrm{X}$, giroversión, hipoplasias de esmalte, fusiones y germinaciones, lo que aumenta la dificultad de la limpieza oral. ${ }^{18}$ El presente estudio no puede evaluar estas anomalías, aun así, enfatizamos la importancia de orientar la higiene, la alimentación, el uso de dentífrico fluorizado para que esa población sea asistida y así evitar problemas futuros. Para tratar anomalías dentarias y tener una buena salud general de la dentición, ${ }^{19}$ es necesario el acompañamiento precoz.

Algunos factores relatados en la literatura que pueden influenciar la calidad de vida de los pacientes fisurados son: las limitaciones funcionales (dificultad en comer, hablar); el dolor y el bienestar emocional (ansiedad).,10,20 Al analizar el cuestionario utilizado (ECOHIS), encontramos estos factores entre las cuestiones englobadas en la sección "Impacto en el niño". Esta sección fue la que presentó la media y la variabilidad mayores al compararla con la sección de "Impacto en la familia". La sección "Impacto en el niño" evalúa 
ítems como: constreñimiento al sonreír; si el niño evita socializar con otras personas; si los mismos faltaron a la escuela o dejaron de hacer alguna actividad por causa de las condiciones bucales; si existe dificultad de alimentación y de pronunciar palabras; o sea, es la sección que evalúa la función, la estética y la fonética, que son los factores más afectados por las fisuras labio palatales. Basados en la información del cuestionario socio comportamental, sobre la dificultad de alimentación, los cuidadores pudieron percibir que este factor puede ser un indicativo por el que la sección "Impacto en el niño" pudo haber presentado un valor medio superior, o sea, estar impactando negativamente.

La sección "Impacto en la familia" evalúa si los cuidadores se sienten culpables por la condición bucal de los niños y si incurrieron en costos altos para cuidar de la salud bucal de los niños. Como el tratamiento realizado en el centro es gratuito y las fisuras labio palatales son malformaciones congénitas que se salen del control de los padres, creemos que este es el motivo por el que no hubo un alto impacto en la calidad de vida con relación a la salud bucal.

El estudio presenta limitaciones como el tamaño de la muestra y falta de datos clínicos para realizar mayores comparaciones debido a la dificultad de conseguir la autorización y el contacto con grandes centros que ofrezcan apoyo a estos pacientes $y$ en este grupo de edades. Sin embargo, tiene relevancia pues es una explotación inicial para investigaciones futuras. Sabiendo del recelo de los cuidadores con relación a la higiene de los pacientes, los profesionales del centro (Afissore) podrán enseñarles cómo realizarla, enfatizando la prevención.

\section{Conclusión}

Se puede concluir que los responsables por los pacientes con fisuras labio palatales presentan dificultad en la limpieza y la alimentación de los niños, así como alteraciones en la calidad de vida con relación a la salud bucal.

\section{Referencias bibliográficas}

1. Mossey Pa, Castillia E. Global Registry And Database On Craniofacial Anomalies. Geneva: World Health Organization; 2003.

2. Al Omari F, Al-Omari Ik. Cleft Lip And Palate In Jordan: Birth Prevalence Rate. Cleft Palate Craniofac J. 2004; 41(6): 609-12.

3. Clarren Sk, Anderson B, Wolf Ls. Feeding Infants With Cleft Lip, Cleft Palate Or Cleft Lip And Palate. Cleft Palate J 1987; 24(3): 244-9.

4. Kramer Fj, Gruber R, Fialka F, Sinikovic B, Schliephake H. Quality Of Life And Family Functioning In Children With Nonsyndromic Orofacial Clefts At Preschool Ages. J Craniofac Surg. 2008; 19: 580-587.

5. Munz Sm, Edwards Sp, Inglehart Mr. Oral Health-Related Quality Of Life, And Satisfaction With Treatment And Treatment Outcomes Of Adolescents/Young Adults With Cleft Lip/Palate: An Exploration. Int J Oral Maxillofac Surg. 2011; 40: 790-796. 6. Hunt, O., D. Burden , P. Hepper , Et Al. The Psychological Effects Of Cleft Lip And Palate: A Systematic Review. Eur J Orthod 2005. 27: 274-285.

7. Who (2003). The World Oral Health Report 2003: Continuous Improvement Of Oral Health In The 21st Century-The Approach Of The Who Global Oral Health Programme. Geneva, Switzerland: World Health Organization [Pubmed] 8. Piovesan C, Antunes Jl, Guedes Rs, Ardenghi Tm. Impact Ofsocioeconomic And Clinical Factors On Child Oral HealthRelatedquality Of Life (Cohrqol).Qual Life Res. 2010; 19: 1359-66. 
9. Antunes Ls, Maues Cpr, Nadaes Mr, Costa Mc, Kuchler Ec, Antunes Laa. The Impact Of Nonsyndromic Oral Cleftson Family Quality Of Life . Spec Care Dentist. 2014; 34(3): 138-43.

10. Bos A, Prahl C. Oral Health-Related Quality Of Life In Dutch Children With Cleft Lip And/Or Palate. Angle Orthod. 2011.

11. Klassen Af, Tsangaris E, Forrest Cr, Wong Kw, Pusic Al, Cano Sj, Syed I, Dua M, Kainth S, Johnson J, Goodacre T. Quality Of Life Of Children Treated For Cleft Lip And/Or Palate: A Systematic Review. J Plastreconstraesthetsurg 2012; 65: 547-57.

12. Tesch, FC; Oliveira, BH; Leão, A. \{Semantic equivalence of the Brazilian version of the Early Childhood Oral Health Impact Scale\}. Cad Saude Publica 2008; 24: 1897-909.

13. Agostini BA, Pinto LT, Koehler M, Emmanuelli B, Piovesan C, Ardenghi TM.Trend of traumatic crown injuries and associated factors in preschool children. Braz Oral Res. 2016 Oct 10; 30(1): e112. doi: 10.1590/1807-3107BOR-2016. vol30.0112.

14. Paul T, Brandt R. Oral And Dental Health Status Of Children With Cleft Lip And/Or Palate. Cleft Palate Craniofac J. 1998; 35 : 329-32.

15. Antonarakis, G. S., P-K. Palaska, and Georges Herzog. Caries prevalence in non-syndromic patients with cleft lip and/or palate: a meta-analysis. Caries research. 2013; 47.(5): 406-13.

16. Moua Am, Andre M, Faraj J, Dias Rb. Avaliação De Bebês Portadores De Fissura Labiopalatina Em Relação Á Higiene Oral. Rev. Odonto. 2009; 17(34): 64-9.

17. Mastrantonio Sds, Castilho Arf, Carrara Cfc. Anomalias Dentárias Em Crianças Com Fissura De Lábio E Palato. Odontol.Clín. Cient. 2009; 8: 273-278.

18. Neves Acc, Patrocínio Mc, Lemek P, \& Ui R T. Anomalias Dentárias Em Pacientes Portadores De Fissuras Labiopalatinas: Revisão De Literatura. Revista Biociências 2008; 8(2).

19. Vallino Ld , Zuker R, Napoli Ja. A Study Of Speech, Language, Hearing, And Dentition In Children With Cleft Lip Only. Cleft Palate-Craniofacial Journal. 2008; 45(5).

20. Topolski Td, Edwards Tc, Patrick Dl. Quality Of Life: How Do Adolescents With Facial Differences Compare With Other Adolescents? Cleft Palate Craniofac J. 2005; 42: 25-32.

Recibido 15/08/2016

Aceptado 11/03/2017

Correspondencia: Carmela Rampazzo Bresolin. carmela_rb@hotmail.com.

Rua Barroso Neto, 420 apto 11. Vila Indiana - Bairro Butantã. São Paulo - SP CEP: 05585-010 Tel: (11) 94860-0084 